\title{
Onco-Fertility Tumor Board is Vitally Important for Addressing Fertility Issues in Cancer Patients
}

\author{
Ahmed Nadeem Abbasi ${ }^{*}$, , Fatima Shaukat ${ }^{1}$, Sehrish Abrar ${ }^{1}$, Bilal Qureshi ${ }^{1}$, Nasir Ali ${ }^{1}$, Asim Hafiz ${ }^{1}$, Hina Hafiz ${ }^{2}$ \\ ${ }^{1}$ Section of Radiation Oncology, Department of Oncology, The Aga Khan University, Karachi, Pakistan. \\ ${ }^{2}$ Department of Medical Oncology, Sindh Institute of Urology \& Transplantation (SIUT), Karachi, Pakistan.
}

Some cancers and some cancer treatments can lead to temporary or permanent infertility. Infertility is a special issue which need to be considered and addressed in cancer patients who may face this issue due to their malignancy itself or due to the treatment offered to them. Our team had reviewed contemporary literature and came to the opinion that it is vitally important to establish a specialized board of experts who can have regular board meetings in which all selected cases of cancer patients are discussed who can potentially suffer from some form of temporary or permanent infertility. The relationship between infertility and cancer is being documented in various studies and systemic reviews published lately $[1,2]$. Counselling of patients and their next of kin is also an important aspect of this proposed multi-disciplinary team (MDT) Board .Before making any consideration regarding the relevance of the issue of infertility in selected cases of cancer the multidisciplinary team has to develop robust processes in order to make sure that we will not be missing this important problem which our cancer patients may face at the time of diagnosis, treatment or later on as a late sequela of oncological treatment. Practice committee on onco-fertility consortium made clear recommendations highlighting the importance of expert team involvement. The proposals are being published in January 2021 issue of Journal of assisted reproduction [3]. This document provides a valuable insight regarding the establishment healthcare quality processes and their practical implications.

This issue of fertility has a special bearing when it comes to the management of pediatric and adolescence cancer patients [4]. Here, we wish to mention the importance of formation of an expert high quality multidisciplinary team to oversee and identify those cases in which fertility issue is relevant. We shared our own views and recommendations in order to over emphasize on this fact [5]. Specialized oncology fertility multi-disciplinary boards can play a pivotal role in the management of these cancer patients. A prospective cohort study conducted in 109 National Health Service (NHS) hospitals across United Kingdom has addressed processes of care and survival associated with treatment in specialist teenage and young adult cancer centers. This study is called BRIGHT-

*Address correspondence to this author at the Section of Radiation Oncology, Department of Oncology, The Aga Khan University, Karachi, Pakistan. Email: nadeem135@gmail.com
LIGHT Cohort Study which is being concluded with the inference of supporting the concept of Principal Treatment Centers care and management. Principal Treatment Centers have multi-disciplinary teams and they showed better management outcomes in Brightlight cohort study [6].

Male and female fertility preservation is an important area of expertise which can only develop with the cooperation and team work of multiple concerned disciplines. Fertility preservation is our cancer patients main concern in many instances. This fact cannot be ignored or undermined that specialist doctors involved in the management of cancer are not always well versed in counselling of these cancer patients. Relying on individual specialist is not advisable. Various options of preservation of fertility can be discussed in this specialized onco-fertility multi-disciplinary board which will be comprised of an expert endocrinology team along with other specialists, e.g., Radiation, Medical, Surgical Oncologists. The issue of fertility preservation will become more and more relevant and important with the passage of time as we are now due to modernization of our oncological interventions, we are getting long term survivors $[7,8]$.

In our teams' endeavors towards the establishment of multidisciplinary culture via working with various other specialties we can vouch on the idea of documenting rare entities and conditions after going through team deliberations in these boards. $[9,10]$. After conducting the multi-disciplinary team board discussions counselling of these patients would be required by appropriately trained specialists. Counselling regarding fertility related issues is recommended before the commencement of any form of oncological treatment [11]. In the presence of a multidisciplinary board this whole process of counselling will get more formalized and it will increase the confidence of patients who are undergoing cancer treatment. Contemporary published literature is strongly recommending Tumor Board establishment. Onco-Fertility Multi-disciplinary Team (MDT) Boards in which all relevant cancer patient cases would be discussed. The cases can be identified beforehand via a steering group which can make a selection criteria selection of cases in which cancer itself or cancer treatment can potentially lead to temporary or permanent male or female infertility. 
The local socio-cultural back ground of our community and culture is not exactly similar when we compare it with the socio-economic and socio-cultural back ground of Western countries. Radiation treatment given to the pelvis in Gynaecological cancer patients in Pakistan also requires pretreatment fertility counseling in selected cases [12]. In our Asian cultures the open discussion on the issue of fertility and reproduction is still considered as a taboo in many instances. Our own published manuscripts confirm this dictum. More work is needed in order to create awareness of patients and to enhance the knowledge of our doctors regarding temporary and permanent infertility caused by cancer or by the treatment of cancer. Therefore we have to create awareness among this section of population who will get oncological management in Low and Middle Income (LMIC) countries [13, 14].

\section{CONFLICT OF INTEREST}

Declared none.

\section{ACKNOWLEDGEMENTS}

Authors are indebted to acknowledge and appreciate the continuous guidance and support received from NJHS-Editorial Manager, Miss Maria Baig. Her expert professional advice at each step of the manuscript preparation journey proved to be a great help for us. Professor Tahir Shamsi and his team is supporting this important patient centered campaign of establishment of site specific multidisciplinary team (MDT) Tumor Boards in Pakistan \& neighboring countries, under the Slogan of " TUMOR BOARDS SAVE LIVES".

\section{REFERENCES}

[1] Zavattaro M, Lanfranco F, Salvagno F, et al. Gonadal failure and infertility in cancer survivors: Clinical management and strategies for prevention. Front Horm Res 2021; 54: 1-11. DOI: $10.1159 / 000515460$

[2] Martinez F, International Society for Fertility Preservation-ESHRE-ASRM Expert Working Group. Update on fertility preservation from the Barcelona International Society for Fertility Preservation-ESHRE-ASRM 2015 expert meeting: Indications, results and future perspectives. Fertil Steril 2017; 108(3): 407-15. DOI: 10.1016/j.fertnstert.2017.05.024

[3] Practice Committee of the Oncofertility Consortium. Installing Oncofertility programs for common cancers in optimum resource settings (Repro-Can-OPEN Study Part II): A committee opinion. J Assist Reprod Genet 2021; 38(1): 163-76. DOI: $10.1007 / \mathrm{s} 10815-020-02012-0$

[4] Burns KC, Hoefgen H, Strine A, Dasgupta R. Fertility preser- vation options in pediatric and adolescent patients with cancer. Cancer 2018; 124: 1867-76. DOI: 10.1002/cncr.31255

[5] Abbasi AN. Establishment \& maintenance of quality of site specific multi-disciplinary tumor boards in Pakistan. J Coll Physicians Surg Pak 2016; 26(10): 805-7.

[6] Fern LA, Taylor RM, Barber J, et al. Processes of care and survival associated with treatment in specialist teenage and young adult cancer centres: Results from the Brightlight cohort study. BMJ Open 2021; 11(4). DOI: 10.1136/bmjopen-2020-044854

[7] Del-Pozo-Lérida S, Salvador C, Martínez-Soler F, Tortosa A, Perucho M, Giménez-Bonafé P. Preservation of fertility in patients with cancer (review). Oncol Rep 2019; 41: 2607-14. DOI: $10.3892 /$ or.2019.7063

[8] Felicetti F, Castiglione A, Biasin E, et al. Effects of treatments on gonadal function in long-term survivors of pediatric hematologic malignancies: A cohort study. Pediatr Blood Cancer 2020; 67(12): e28709. DOI: 10.1002/pbc.28709

[9] Lateef N, Abdul Basit K, Abbasi N, Kazmi SM, Ansari AB, Shah M. Malignancies After heart transplant. Exp Clin Transplant 2016; 14(1): 12-6.

[10] Khan BM, Mansha MA, Ali N, Abbasi AN, Ahmed SM, Qureshi BM. Hidradenocarcinoma: Five years of local and systemic control of a rare sweat gland neoplasm with nodal metastasis. Cureus 2018; 10(6): e2884.

[11] Bentsen L, Pappot H, Hjerming M, Colmorn LB, Macklon KT, Hanghøj S. How do young women with cancer experience oncofertility counselling during cancer treatment? A qualitative, single centre study at a Danish Tertiary Hospital. Cancers (Basel) 2021; 13(6): 1355. DOI: 10.3390/cancers13061355

[12] Ali N, Valimohammad AT, Abbasi AN, Mansha MA, Hafiz A, Qureshi BM. Chemoradiation and the role of adjuvant chemotherapy in lymph nodal-metastatic cervical cancer. J Glob Oncol 2018; 4: 1-4. DOI: 10.1200/JGO.2017.009852

[13] Osmani AH, Haider G, Ali S, Ali F, Irfan M, Fatima DE. Knowledge and perceptions about cancer treatment-associated infertility among young patients at a tertiary care hospital in Pakistan. Asian Pac J Cancer Prev 2017; 18(12): 3261-5.

[14] Idrees R, Fatima S, Abdul-Ghafar J, Raheem A, Ahmad Z. Cancer prevalence in Pakistan: Meta-analysis of various published studies to determine variation in cancer figures resulting from marked population heterogeneity in different parts of the country. World J Surg Oncol 2018; 16(1): 129. PMID: 29976196; PMCID: PMC6034324. DOI: 10.1186/s12957-018-1429-Z 